\title{
Load balancing ad hoc on-demand multipath distance vector (LBAOMDV) routing protocol
}

\author{
Saleh A. Alghamdi
}

\begin{abstract}
Researchers working in the area of a mobile ad hoc network (MANET) strive to conserve the battery energy of individual nodes to reduce the frequency of a node breakdown. The model of multiple-path on demand data routing protocols has been an effective scheme for the majority of MANET application scenarios in recent times. The availability of multiple paths for data transfer can both prove to be effective as well as dismal in certain cases. The selection of the most suitable path is always tricky, if not associated with exact metrics of concern. The contribution of this work is the introduction of the load balancing ad hoc on-demand multipath distance vector (LBAOMDV) protocol, an adaptation of AOMDV, an ad hoc on-demand multipath distance vector protocol. The adaption is done in order to enhance the reliability of the given network by considering the parameter of path weight (energy) of all the available multiple paths. The LBAOMDV regulates the fair usage of both node energy and available bandwidth by exploiting the availability of multiple paths for data transfer. The uniform distribution of data across multiple paths enhances the quality of service of the given network by ensuring fair usage of both network bandwidth and node energy. The $\angle B A O M D V$ protocol ensures reduced node breakdowns, thus enhancing the reliability of the given MANET.
\end{abstract}

Keywords: MANET; AODV; AOMDV; LBAOMDV; Load balancing; Fault tolerance

\section{Introduction}

A mobile ad hoc network (MANET) is a collection of wireless mobile nodes which dynamically form a wireless network without a fixed infrastructure or a wired backbone network, as shown in Fig. 1. A mobile ad hoc network is a multi-hop network without any predetermined topology or central control, hence making routing a crucial design issue for these networks [1]. One of the most important and thoroughly discussed areas in wireless communication has been channel assignment and usage. As ad hoc networks are decentralized, they require advanced bandwidth allocation schemes [2]. Various issues ranging from topology control and network coverage to channel assignment have been addressed recently, and viable solutions have come forth. The issue of topology control and streamlined data communication has been thoroughly discussed $[3,4]$. The advent of wireless technology in the public domain has

\section{Correspondence: salehatiah@bu.edu.sa}

Department of Computer Engineering, Al-Baha University, Al-Baha, Saudi Arabia raised issues related to network coverage for reliable communication [5-7]. Recently, a new area of concern for researchers has emerged known as green mobile networks, which are wireless networks sensitive to energy usage and dissipations [8].

The rapid node addition or departure frequency of wireless nodes adds to the complexity of routing in MANETs. The inherent issue of rapid mobility complicates the issue further, by making it difficult to address a particular topology for a long [9-11]. The fundamental concern related to the complexity of routing has been addressed, but still some inherent bottlenecks are unresolved. The issue related to delay in route selection has been discussed, and many viable solutions have been proposed in the past $[12,13]$. The issues related to congestion and its impact on data delivery have also been addressed [14]. In the past, the basic principle of routing protocols generally was limited to identifying a single path between source and destination node, with a constraint of minimum hop counts. This genre of routing

\section{Springer}




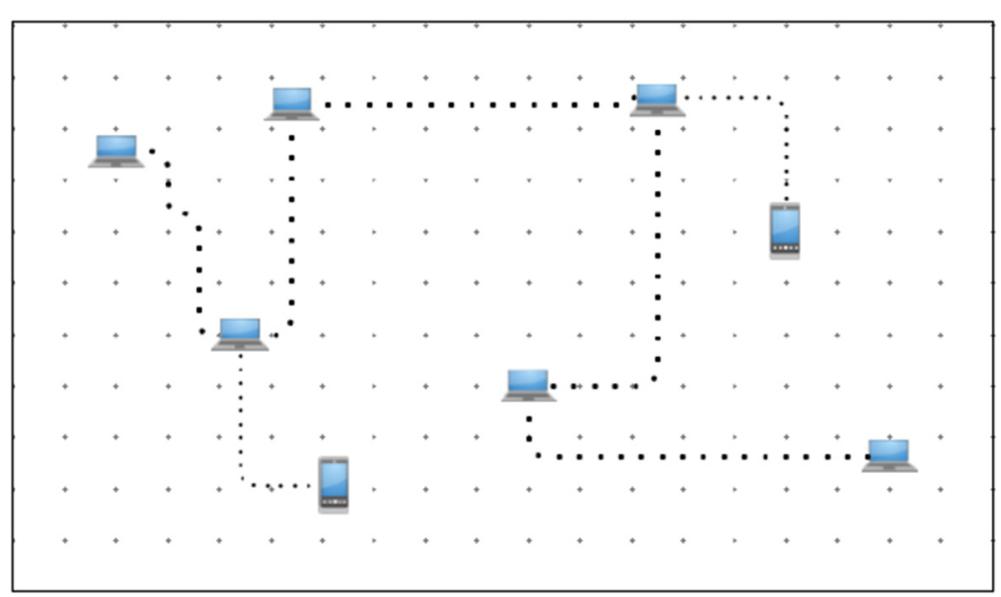

Fig. 1 A basic mobile ad hoc network (MANET)

protocols had limitations of reliability and bandwidth usage owing to path breakdown. Nevertheless, in a reasonably well-connected network, there exists several paths between a source node and a destination node. Multipath routing is one of the most effective trends in the area of wireless routing [15]. The concept of multipath routing involves discovering all available paths from the source node to the destination by taking advantage of path redundancy of the underlying network. These multiple available paths may be used alternately or in certain scenarios concurrently for data transfer $[16,17]$.

The process of routing both in general computer networks, as well as in wireless networks, can be reactive or proactive. The category of reactive routing protocols has been the most effective for wireless scenarios, and the introduction of reactive multipath on demand protocols has steered to a new generation of MANET routing. The most rigorously discussed reactive wireless (on-demand) routing protocols to date have been Dynamic Source Routing (DSR), Ad hoc On-Demand Distance Vector Routing $(A O D V)$ and Temporally Ordered Routing Algorithm (TORA) [18-20]. All these reactive routing protocols flood a network with route request packets (RREQ) to discover and maintain routes, only after receiving a request for data transfer. Proactive routing protocols such as Destination Sequenced Distance Vector Routing $(D S D V)$ or other DSDV variants tend to discover and maintain routes between all pairs of nodes in advance to any data requests [21]. The application of proactive protocols seems unrealistic for mobile wireless scenarios, as the frequent topology changes, that may require frequent updating of routing tables which will incur huge routing overhead [22].

The transfer of data over a single path among the various paths has been the principle of the most on-demand multiple path routing schemes. If the route fails again, a new route is evaluated from source to destination, consuming time and resources. So it is obvious that distributing data packets among the discovered multipaths is the key solution. The research in the recent past has resulted in some advanced techniques for routing in wireless communication networks [23]. Advanced areas of data communication in wireless sensor networks have been taken both by the academia and industry and have yielded significant results [24, 25]. Techniques addressing the issues related to sensor networks for data processing and delivery have also been part of recent research developments in wireless technology [26-28]. The concept of information-centric networks (ICN) has also come into light during the last half decade. These information-centric networks pose quite a different set of challenges [29]. Advances in making IoT a successful technology in future requires greater integration between existing network platforms and the internet, thus enabling their ability to provide services across the globe [30].

The work through this paper mainly concentrates on effectively balancing the data load among the discovered multipaths in order to maintain nodes resources and reduce the traffic to avoid creating traffic load or bottlenecks.

This paper is organized as follows. Section 2 covers related work in the area of on-demand multipath routing protocols. Section 3 proposes the LBAOMDV load-balanced ad hoc on-demand multipath distance vector routing protocol. Section 4 provides the simulation details of the environment set for the NS2 network simulator. Section 5 of this paper discusses in detail the results generated from simulation of the proposed $L B A O M D V$ protocol.

\section{Background}

Many conventional routing protocols developed in the past have least emphasized the optimum utilization of 
network bandwidth and node energy [31]. But lately, several multipath routing protocols have targeted the energy consumption issues while devising routing schemes for wireless mobile networks [32]. A detailed discussion of the advances in mobile ad hoc routing is provided in this section.

Effective load balancing of data across multiple available paths has been utilized for fair bandwidth usage in mobile ad hoc networks [33]. Pearlman et al. proposed an alternate path routing $(A P R)$ that provided load balancing by distributing traffic among a set of diverse paths. APR is an ideal candidate for mobile ad hoc networks with limited channel bandwidth [34]. Yin et al. introduced a load balancing technique called multipath adaptive load balancing (MALB). MALB dynamically distributes the traffic among multiple paths, based on path statistics measurement. $M A L B$ is an inclusive framework and can collaborate with any kind of multipath source routing protocols [35]. Mérindol et al. had proposed a scheme that offers the possibility to use temporarily alternative routes in order to reduce packet loss and degraded throughput [36]. Nagarjun et al. proposed a Packet Count-Based Routing Mechanism (PCRM) protocol. PCRM is based on Dynamic Source Routing (DSR) protocol. In contrary of all other regular load balancing techniques, PCRM selects the least used path for sending data packets rather than the mostly used one that regularly includes the minimum hop count [37]. Sharma et al. proposed a similar solution to utilize available bandwidth of the channel multiple disjoint paths. The approximation of bandwidth of a given path is done by sending detector packets across a network. The source node chooses the path with maximum bandwidth as the primary route for forwarding data [38]. Recently, Qi et al. proposed a multipath routing protocol (EM$A O D V$ ), based on $A O D V$. EM-AODV evaluates the paths using the parameter values of path energy and hop counts [39]. The advancements in AODV have resulted in more efficient protocols like ad hoc on-demand multipath distance vector $(A O M D V)$ that is more suitable for MANET scenarios [40]. Advancements in the routing techniques in wireless communication have also been based on various intelligent techniques. Athanasios et al. introduced an intelligent approach to improve quality of service $(Q o S)$ routing in ATM networks [41]. Thrasyvoulos et al. in their work provided insights into design of routing algorithms for vehicular ad hoc networks [42]. Moustafa et al. described the routing metrics for routing in cognitive radio networks in detail [43]. The designing of vehicular networks is also quite challenging owing to rapid mobility. Few techniques to enhance the functionality of vehicular networks are being floated for discussion in the industry and academia [44, 45]. The latest advancements in improving the quality of routing has been studied by researchers, and their findings have uncovered new challenges [46]. The most interesting solution for these kinds of challenges in dynamic routing has been the efficient multipath routing schemes [47]. Some recent work carried out in the direction of QoS, the quality of service constrained efficient path selection has been noteworthy [48-50]. The applications of basic network concepts have been realized in other spheres of science and technology as well. These realizations have also been incorporated into wireless networks to achieve significant gains in efficient routing [51,52]. The current work is in continuation of the previous established attempts and has shown significant improvement in the same direction. The current work has utilized the popular NS2 network simulator for deriving and showcasing the results [53]. In the next section, we propose the $L B A O M D V$ protocol that takes QoS to a higher level by enhancing both performance and reliability of MANETs.

\section{Load balancing ad hoc on-demand multipath distance vector (LBAOMDV) protocol}

A MANET can be realized by an undirected graph $G(V, E)$, where $V$ is the number of nodes and $E$ is the total possible edges. Here, we associate a definite weight representing the amount of energy that gets consumed for the transfer of a single fixed length data packet between a pair of nodes. So, each edge in the given network graph will have a weight $\mathrm{WE}_{i j}$ representing the amount of energy consumed for a single hop transfer from node $i$ to node $j$. The amount of energy for a single hop transfer between the given two network nodes can be calculated by a trivial function given in Equation (1):

$$
\mathrm{WE}_{i j}=E\left(V_{i}, V_{j}\right)
$$

The set of $\mathrm{WE}_{i j}$ values for all the possibilities of edges of the given network graph $G(V, E)$ can be represented by a weighted adjacency matrix depicted in Fig. 2.

The value of the weight $\mathrm{WE}_{i j}$ of a given edge may be zero, in case a direct link is not available between a node pair $V_{i j}$. A given path " $P_{i j}$ " from a source node $V_{i}$ to a destination node $V_{j}$ can be comprised of $n$ nodes and $n$ -1 multiple hops where $1 \leq n \leq V$.

The amount of energy required for the transfer of a single data packet from $V_{i}$ to $V_{j}$ can be calculated by the summation of weights $\mathrm{WE}_{i j}$ of all participating hops and can be given by Equation (2).

$$
\mathrm{PE}_{i j}=\sum_{i=1}^{n-1} E\left(W_{i}\right)
$$

The value $\mathrm{PE}_{i j}$ represents the amount of energy consumed in the transfer of a single packet of data along the given path $P_{i j}$. A message " $M$ " to be transferred from a source to destination node can have " $Q$ " number of packets. So, the amount of energy " $€$ " required for 


\begin{tabular}{|c|c|c|c|c|c|c|}
\hline & 1 & 2 & 3 & ......... & V-1 & V \\
\hline 1 & 0 & $W_{12}$ & $W_{13}$ & ......... & $W E_{1, j-1}$ & $W E_{1 j}$ \\
\hline 2 & $W_{21}$ & 0 & $W_{23}$ & ......... & $W E_{2, j-1}$ & $W_{2 j}$ \\
\hline 3 & $W_{31}$ & $W_{32}$ & 0 & ........ & $W E_{3, j-1}$ & $W E_{3 j}$ \\
\hline ......... & ......... & ......... & ........ & 0 & ........ & .......... \\
\hline V-1 & $W E_{i-1,1}$ & $W E_{i-1,2}$ & $W E_{i-1,3}$ & ......... & 0 & $W E_{i-1, j}$ \\
\hline V & $W_{E_{i 1}}$ & $W_{E_{i 2}}$ & $W E_{i 3}$ & ......... & $W E_{i, j-1}$ & 0 \\
\hline
\end{tabular}

Fig. 2 Weighted adjacency energy matrix

transferring a message " $M$ " from source to destination node along a given path $P_{i j}$ can be calculated as given in Equation (3).

$$
£=Q\left(\mathrm{PE}_{i j}\right)
$$

A message $M$ comprising of $Q$ packets of data can be divided into fixed $C$-sized chunks of data to be distributed among $K$ available multiple channels as given in Equation (4).

$$
C=M / K
$$

The load balancing of data across multiple channels ensures optimum utilization of the available bandwidth for a given wireless network. A path $P_{i j}$ is a sequence of nodes and links from the intended sender to the intended receiver. The efficiency of data transfer among a node pair primarily depends on node capacity $L$ and link traffic $T$ of a given data channel. A protocol is said to have a fair bandwidth usage if it allows balanced traffic across the available paths. The amount of unused bandwidth $(U B)$ is a firm indicator for ensuring equal usage of bandwidth across different paths. The UB parameter for a node pair or hop provides us the value of unused bandwidth at the particular hop and can be derived as given in Equation (5).

$$
\mathrm{UB}=L-T
$$

To ensure that the node with minimum unused bandwidth (MUB) over a path $P_{i j}$ can handle the allocated data chunk, it can be derived from Equation (6).

$$
\mathrm{MUB}_{i j}=\min _{i=1 \text { to } j}(\mathrm{UB})
$$

\subsection{Dynamic load balancing}

The abrupt increase in the non-uniform requests from individual network nodes for data has lead to a burst mode of data transfer across network channels. This burst mode of network data transfer has increased the importance of reliability assessment at peak times. The challenges of increasing the reliability of a given network has been associated to the two significant factors, namely node energy and channel bandwidth. By transferring data across multiple paths, the $L B A O M D V$ protocol addresses both these factors of bandwidth and node energy.

Table 1 Simulation details

\begin{tabular}{ll}
\hline Simulator & NS-2.35 \\
Routing protocols & $\begin{array}{l}\text { LBAOMDV, AOMDV, PCRM, } \\
\text { and AntHocNet }\end{array}$ \\
Various simulation time spaces (s) & $50,100,200,400,1000$ \\
Simulation area & $1000 \mathrm{~m}$ *1000 m \\
Various network sizes (node number) & $25,50,100,200$ \\
Transmission range (m) & 250 \\
Mobility model & Random way point \\
Maximum speed & $5 \mathrm{~m} / \mathrm{s}$ \\
Data packet size & $512 \mathrm{~KB}$ \\
Traffic source CBR & Traffic source CBR \\
Initial energy of participating & $40 \mathrm{~J}$ \\
network nodes & \\
\hline
\end{tabular}


The basic path finding technique given in the $A O M D V$ protocol is used exactly in the $L B A O M D V$ protocol based on the flooding of route request packets (RREQ) across the whole network. Each reverse route is established on reception of positive route reply packet $(R R E P)$. A set of multiple disjoint paths are established by interleaved exchange of the $R R E Q$ and $R R E P$ route establishing packets [20]. Among these multiple discovered routes or paths, the final set of paths for actual data transfer is selected based on effective energy utilization assessment. The criterion for path selection is based on the amount of energy to be consumed over a path for transfer of data chunk $C$. A path $P_{i j}$ belongs to the set of paths for final data transfer if the value of $P E$ is greater than the amount of energy required for each chunk transfer, as given in Equation (7).

$$
\mathrm{PE}_{i j} \geq £ / K-1
$$

Where $K$ is the number of discovered paths, after the route discovery session gets completed. The selection of paths on the condition given in Equation (7) allows balanced energy utilization of nodes in a given network. After the selection of paths, the qualified paths are arranged based on the average unused bandwidth (PUB) of a particular path $P_{i j}$. This arrangement ensures that paths are used in the descending order of value for $\mathrm{PUB}_{i j}$.

The next phase of the $L B A O M D V$ protocol ensures actual data transfer over the qualified multiple channels. A complete message is divided into fixed-sized chunks which are transferred over the multiple qualified paths. A successful data transfer of a complete

\section{Start-of- $L B A O M D V$ Algorithm}

\section{Phase1. Route Discovery}

Step1. Flooding route request RREQ messages seeking for the destination.

Step2. RREQ propagation from the source node to the destination node establishes multiple reverse paths both at intermediate nodes as well as the destination.

Step3. Multiple RREPs traverse through these reverse paths back, to form multiple forward paths to the destination at the source and intermediate nodes.

\section{Phase2. Load Balancing}

Step4. For all, discovered routes $P_{i j}$

If $\left(P E_{i j}>=£ / K\right)$

Add $P_{i j}$ to the qualified list List[] of paths, for data transfer.

Step5. Arrange the qualified list List[], in descending order of $M U B_{i j}$.

\section{Phase3. Actual Data Transfer}

Step6. Transfer all the C sized chunks of data over qualified list of paths.

\section{Step7. If (Path-Fails)}

$$
\begin{gathered}
M=C_{f} \\
K=K-1
\end{gathered}
$$

$$
\text { /* Assign Failed data chunk } C_{f} \text { as a new message } M * /
$$$$
\text { /* One Qualified path fails */ }
$$

\section{Goto Step2.}

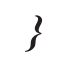

Step8. Exit (1);

\section{End-of-Algorithm.}




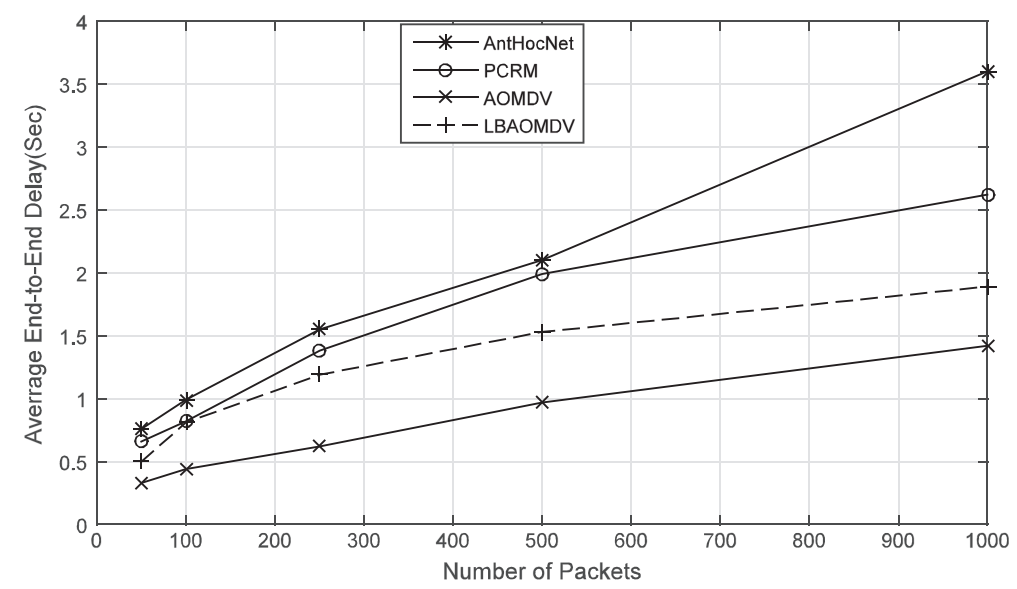

Fig. 3 Average end-to-end delay vs. no. of packets

message will result in the end of the $L B A O M D V$ protocol process. An unsuccessful data transfer due to path breakdown will require that the failed data chunk be retransferred. This retransfer of data will be treated as a new message and will be distributed across all the $K-1$ available channels.

\subsection{LBAOMDV algorithm}

The implementation of LBAOMDV protocol is divided into three phases.

\subsection{Reliability assessment of the LBAOMDV Protocol}

In a load-balanced multipath routing environment, a message is decomposed into equal-sized data chunks and each such data chunk is transferred over a single path to its intended destination. Here, we assume that " $K$ " number of available multiple paths are both disjoint (node and link) and non-cyclic for the current discussion. An intermediate node " $n$ " which is part of ith disjoint path " $K_{i}$ " may fail due to any of the reasons like exhausting energy below the given energy threshold, malfunctioning of any node component, physical damage, or any another correlated reasons. Here, we define three node failure types based on the consequences a node breakdown may have on an ongoing data transmission.

Type 1 failure: A breakdown of an intermediate network node which will halt current ongoing data transfer and initiates a retransmission of the same data chuck over the rest of the alternate available paths, as proposed in the $L B A O M D V$ algorithm.

Type 2 failure: A breakdown of an intermediate network node which does not belong to any of the multiple possible paths for the current data transmission; thus, it will not affect the ongoing data transfer over the network.

Type 3 failure: A breakdown of source/destination node or both or a breakdown of multiple network nodes at the same time, thus affecting the overall functionality of the whole network.

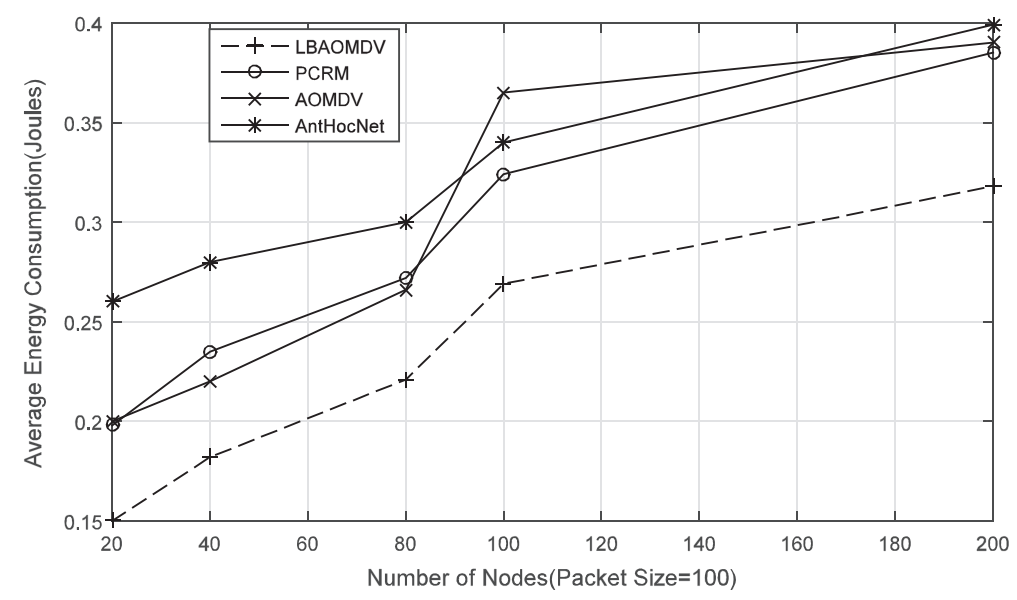

Fig. 4 Average node energy consumption vs. no. of nodes for 100-packet size 


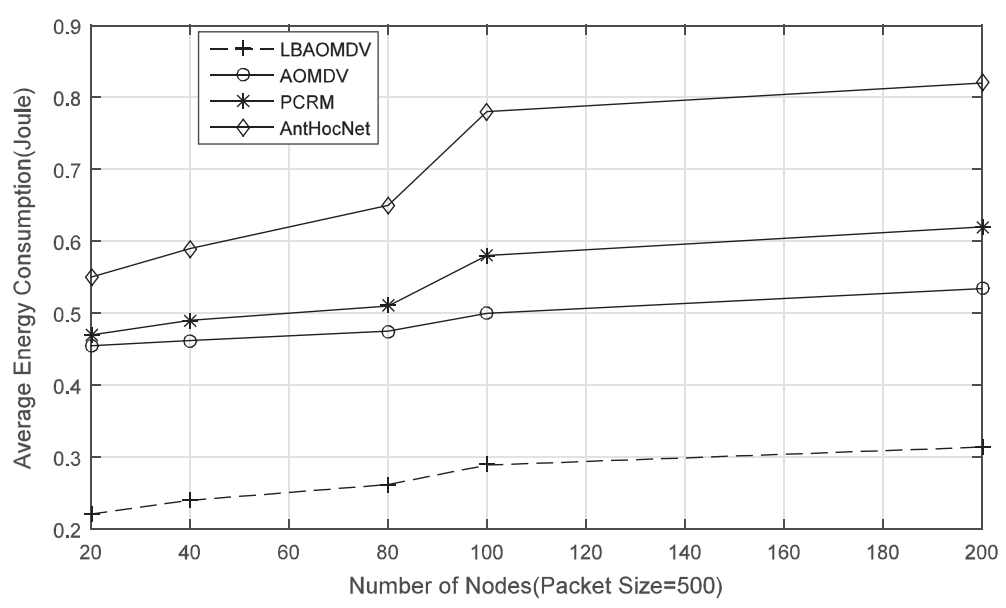

Fig. 5 Average node energy consumption vs. no. of nodes for 500-packet size

Here, in this work, we will be limited to only type 1 failures, and the mention of the term failure in the subsequent discussion will be taken as type 1 failure.

The reliability assessment of the proposed $L B A O M D V$ protocol is done on the mean time between failure $(M T B F)$ metric of reliability evaluation. Here, we define the $M T B F$ of a given MANET as the average number of data retransmissions required during the whole span of data transmission given by Equation (8).

$$
\operatorname{MTBF}=\left[\sum_{i=1}^{n} f(t)\right] / n
$$

Where $n$ is the number of retransmissions and $f(t)$ is the failure time. The mean time to repair (MTTR) metric is defined as the additional delay (time) imposed due to the rerouting and retransmission of the failed data chunk given by Equation (9).

$$
\operatorname{MTTR}=r(t)+\lambda
$$

The results for the reliability and related improvements in energy and delay are discussed in following section of this paper.

\section{Simulation and results of the LBAOMDV protocol}

\subsection{Performance metrics}

The performance of the $L B A O M D V$ protocol is evaluated with respect to existing standard routing schemes like AOMDV, PCRM, and AntHocNet for measuring its performance for standard QOS metric parameters and reliability. $L B A O M D V$ is an advanced version of the $A O M D V$ scheme; the comparison is done to show the improvements achieved. PCRM and AntHocNet schemes are also simulated for comparison with the $L B A O M D V$, as the former two protocols have been quite successful

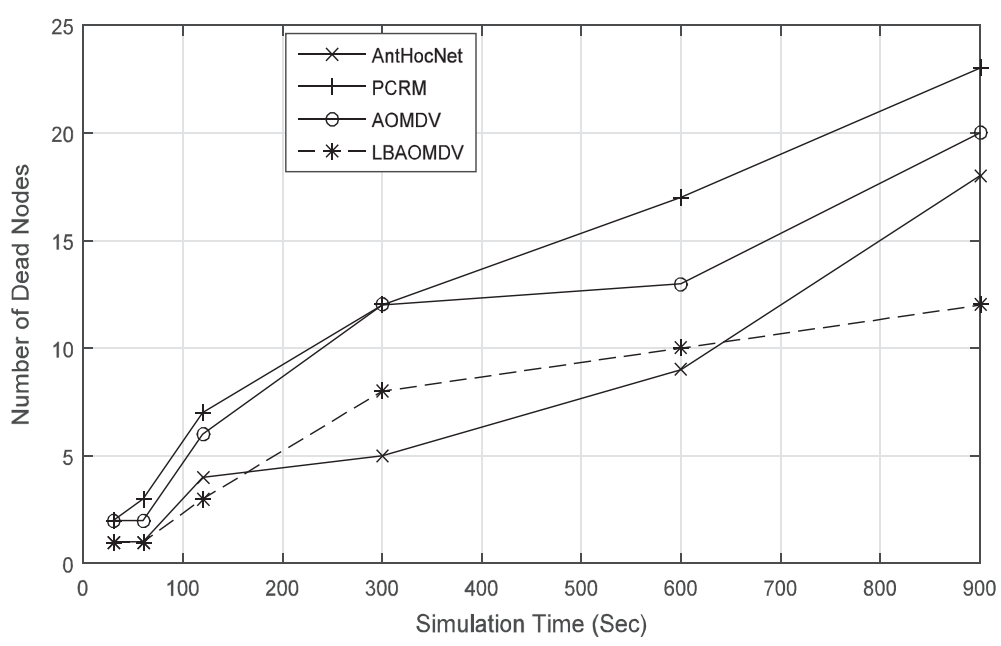

Fig. 6 Number of dead nodes vs. simulation time 


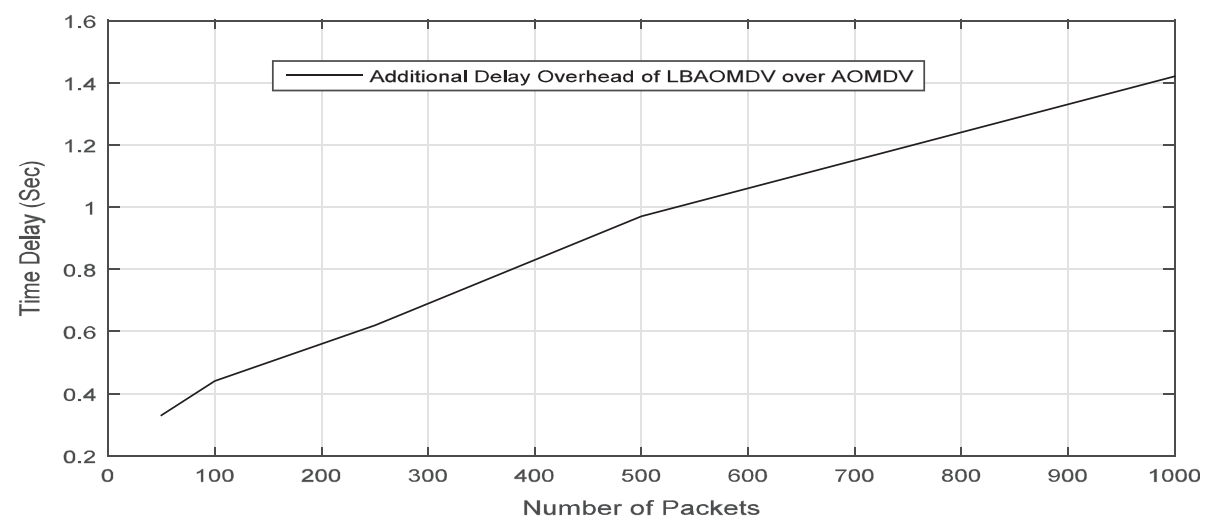

Fig. 7 Additional overhead of LBAOMDV

in wireless ad hoc scenarios. The evaluation metrics are given in the following listing:

1. End-to-end delay: the average delay of data packets from a source to a destination

2. Average energy consumption: the average energy consumed by all nodes in the network

3. Number of dead nodes: the number of node breakdowns during complete data transfer simulations

\subsection{Simulation environment}

The proposed $L B A O M D V$ protocol was simulated, and its performance was evaluated against $A O M D V, P C R M$, and AntHocNet protocols using the prominent NS2 network simulator. Table 1 shows the details of the simulation environment features.

\subsection{End-to-end delay}

The study of delay associated with the LBAOMDV protocol can be assessed by the comparison of the endto-end delays of the data packets transferred over a network using AOMDV, PCRM, and AntHocNet. The comparison of the average end-to-end delays versus packet number is shown in Fig. 3. The proposed $L B A O M D V$ showed higher average end-to-end delays than $A O M D V$, while showing better results compared to PCRM and AntHocNet. The average end-to-end delay for 50 packets is $0.83 \mathrm{~s}$ for $\angle B A O M D V$ while for $A O M D V$ is $0.5 \mathrm{~s}$. While at 1000 packets, $L B A O M D V$ is over AOMDV by nearly $1.2 \mathrm{~s}$ for end-to-end delays. It is also obvious that the increasing rate of average end-toend delay of $L B A O M D V$ is much higher than $A O M D V$, the reason being additional computation required for load balancing. The increase in end-to-end delay in $L B A O M D V$ requires a trade-off with the efficiency achieved by the protocol in terms of efficient bandwidth and energy usage. A real-time network application may require minimum end-to-end data delay, while as nonreal-time applications may be concerned about efficient bandwidth and energy usage.

\subsection{Average energy consumption}

The study of energy consumption of individual nodes across the whole MANET can be assessed by the average energy consumption of the participating nodes after the

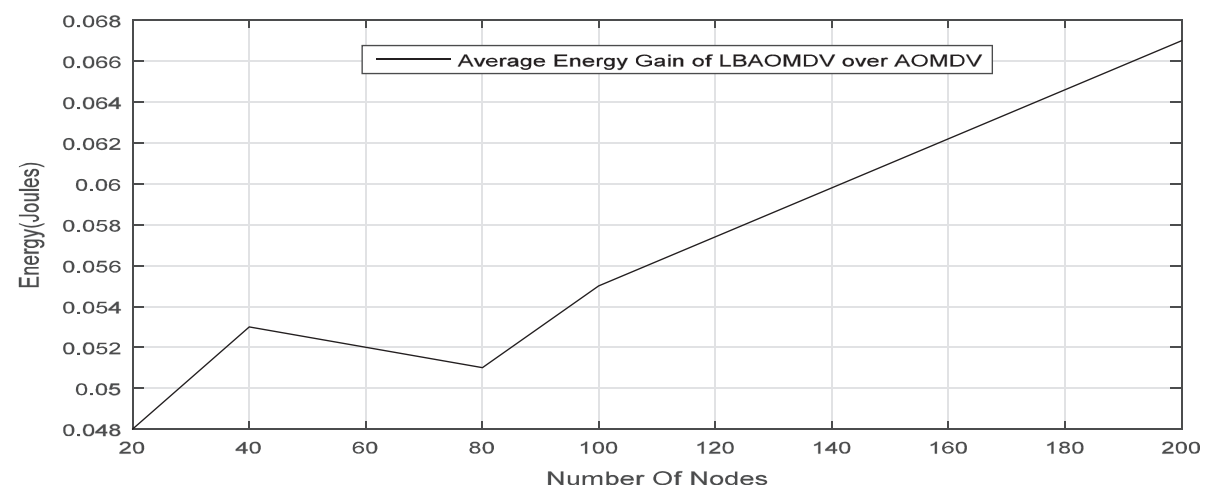

Fig. 8 Average energy gain vs. node size 


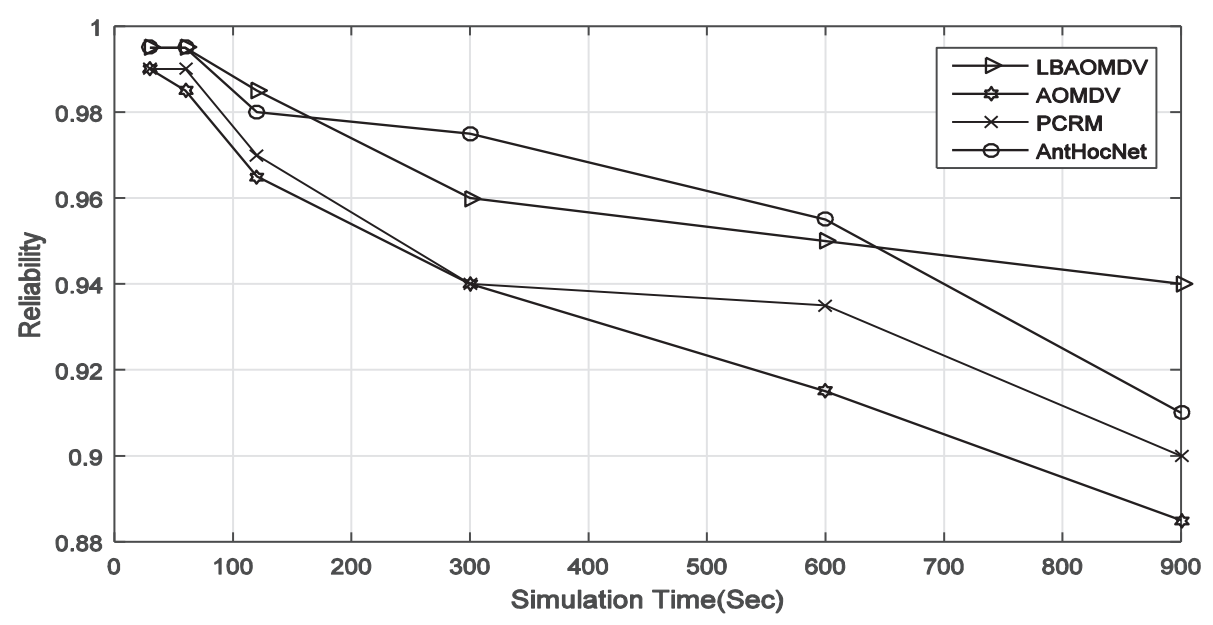

Fig. 9 Reliability curve comparison for LBAOMDV

data is transferred over a network using various standard protocols like AOMDV, PCRM, and AntHocNet in comparison to $L B A O M D V$. Here, energy consumption was tested in two different scenarios of 100 and 500 packets, while each packet was having a size of $512 \mathrm{~KB}$. This can be noticed from Fig. 4 that the performance of LBMODV is much better with lower data loads of 100 packets in this case. The comparison for the average energy consumption of nodes shows improvement in LBAOMDV compared to AOMDV, PCRM, and AntHocNet. While Fig. 5 provides a lucid picture for 500 packets, the $L B A O M D V$ achieved a real improvement as compared to AOMDV, PCRM, and AntHocNet, for the consumed energy which was one of the objectives of this work. For example, at 200 nodes $L B A O M D V$ consumes 0.31 while $A O M D V$ scores 0.53 thus signifying that the network and data load size is a factor in increased energy consumption.

\subsection{Number of dead nodes}

The increasing number of dead nodes causes a double jeopardy to the MANET as it causes data loss and network partitioning. One of the objectives of $L B A O M D V$ is the drastic decrease in the frequency of node breakdowns. Figure 6 shows a less number of dead nodes while simulating $L B A O M D V$ as compared to $A O M D V$, PCRM, and AntHocNet. A $900 \mathrm{~s}$ of simulation showed that $L B A O M D V$ resulted in a drastic decrease for the number of dead nodes (35 out of 200 nodes) which nearly equals half of the number of dead nodes resulted while using $A O M D V$ (64 out of 200 nodes).

Further, it can be concluded that $L B A O M D V$ performed better than AOMDV, PCRM, and AntHocNet in reducing the energy consumption and frequency of node breakdowns (dead nodes). LBAOMDV has clearly showed superiority making it as a better alternative than existing standard multipath ad hoc on-demand routing protocols.

\subsection{Reliability analysis}

Assuming that the time of data transmission for a fixedsized data remains constant across all the multiple available paths and is denoted here by " $\lambda$ ". The amount of additional overhead imposed by $L B A O M D V$ as compared to $A O M D V$, due to the load balancing phase for data rerouting, is shown in Fig. 7. Thus, simulation results generated for the average energy gain of a wireless mobile ad hoc network shown in Fig. 8 show the improvements achieved by the proposed $L B A O M D V$ protocol. The possible improvements in average energy consumption and degradation in the number of dead nodes has resulted in the significant improvement in the reliability of the given network. These improvements in average node energy and node lifetime consequently impact on general reliability of the given wireless network as can be seen in Fig. 9 .

\section{Conclusion}

A multipath routing protocol needs to be governed by a load balancing scheme in order to escape from devouring the energy of participating nodes. Unbalanced battery energy consumption may lead to node breakdown consequently affecting the reliability of the given network in general. The objective of this research work was to develop a bandwidth and energy-aware multipath ondemand routing protocol for MANETs. The proposed $L B A O M D V$ protocol achieves the objective by load balancing of data across multiple available paths. The $L B A O M D V$ protocol further ensures balanced retransmission of the data, in case of any path or node breakdown during a data transmission. The results generated from the simulation for node breakdown and average 
energy consumption parameters showed the improvements achieved by $L B A O M D V$ in comparison to $A O M D V, P C R M$, and AntHocNet. These results conclude to the fact that the $L B A O M D V$ protocol enhances the reliability of a given $M A N E T$ and ensures better network performance at peak data transfer times. This work requires further enhancements in future so as to improve the computational efficiency of load balancing.

\section{Competing interests}

The authors declare that they have no competing interests.

Received: 16 May 2015 Accepted: 16 September 2015

Published online: 06 November 2015

\section{References}

1. S. Corson, J. Macker, Mobile ad hoc networking (MANET): routing protocol performance issues and evaluation considerations, RFC2501, Naval Research Laboratory, University of Maryland, (1999).

2. C Hongju, Xiong, AV Vasilakos, Y Laurence Tianruo, C Guolong, Z Xiaofang, Nodes organization for channel assignment with topology preservation in multiradio wireless mesh networks. Ad Hoc Networks 10(5), 60773 (2012).

3. $L$ Mo, $L$ Zhenjiang, AV Vasilakos, A survey on topology control in wireless sensor networks: taxonomy, comparative study, and open issues. Proc. IEEE 101(12), 25382557 (2013)

4. M. Reza. Rahimi, Nalini, Venkatasubramania, MAPCloud: mobile applications on an elastic and scalable 2tier cloud architecture, IEEE/ACM UCC (2012).

5. Yanjun Yao; Qing Cao; Vasilakos, A.V, EDAL: An energy efficient delay aware, and lifetime balancing data collection protocol for wireless sensor networks. MASS (2013), IEEE International Conference, 182-190.

6. S Yuning, L Liang, M Huadong, AV Vasilakos, A biology based algorithm to minimal exposure problem of wireless sensor networks. IEEE Trans. Netw. Serv. Manag. 11(3), 417430 (2014).

7. S Sengupta, S Das, M Nasir, AV Vasilakos, W Pedrycz, An evolutionary multi objective sleep scheduling scheme for differentiated coverage in wireless sensor networks. IEEE Trans Syst Man Cybern Part C 42(6), 10931102 (2012).

8. W Xiaofei, AV Vasilakos, C Min, L Yunhao, K Ted Taekyoung, A survey of green mobile networks: opportunities and challenges. MONET 17(1), 4-20 (2012).

9. Niranjan Potnis, Atulya Mahajan, Mobility models for vehicular ad hoc network simulations, Proceedings of the 44th annual Southeast regional conference, ACM New York, Melbourne, Florida, (2006).

10. E. Royer and C-K. Toh, A review of current routing protocols for ad hoc mobile wireless networks, IEEE Journal Personal Communications, 6(2), 4655, (1999).

11. S Marwaha, D Srinivasan, CK Tham, A Vasilakos, Evolutionary fuzzy multi objective routing for wireless mobile ad hoc networks, Evolutionary Computation, in Proceedings of the 2004 Congress on Evolutionary Computation (CEC '04), 2 1964-1971, (2004).

12. D Der-Rong, J Jhong-Yan, Delay-constrained survivable multicast routing problem in WDM networks. Comput Commun 35(10), 1172-1184 (2012).

13. Z Xin Ming, Z Yue, Y Fan, AV Vasilakos, Interference-based topology control algorithm for delay-constrained mobile ad hoc networks, in mobile computing. IEEE Trans 14(4), 742-754 (2015).

14. C Busch, R Kannan, AV Vasilakos, Approximating congestion + dilation in networks via "quality of routing" games. IEEE Trans. Computers 61(9), $12701283(2012)$

15. S Mueller, RP Tsang, D Ghosal, Multipath routing in mobile ad hoc networks: issues and challenges, in Performance tools and applications to networked systems, vol. 2965 of lecture notes in computer science (Springer Berlin, Germany, 2004), 209-234.

16. Peng Li; Song Guo; Shui Yu; Vasilakos, A.V., CodePipe: an opportunistic feeding and routing protocol for reliable multicast with pipelined network coding, in INFOCOM, 2012 Proceedings IEEE, 100-108, 25-30 (2012).

17. W Lou, W Liu, Y Zhang, Performance optimization using multipath routing in mobile ad hoc and wireless sensor networks. Combinator. Optim. Commun. Netw. 2, 117-146 (2006).
18. L Peng, G Song, Y Shui, AV Vasilakos, Reliable multicast with pipelined network coding using opportunistic feeding and routing. IEEE Trans Parallel Distrib Syst 25(12), 32643273 (2014)

19. R. N. Noorani, Comparative analysis of reactive MANET routing protocols under the traffic of TCP VEGAS with mobility considerations, International Conference on Emerging Technologies, 457-461, 19-20 Oct. (2009).

20. D. Johnson and D. Maltz., Dynamic source routing in ad hoc wireless networks, In Mobile Computing book, The Kluwer International Series in Engineering and Computer Science, Springer USA, 353 153-181, (1996).

21. C. E. Perkins and E. M. Royer, Ad hoc on-demand distance-vector routing, In IEEE Workshop on Mobile Computing Systems and Applications, Louisiana, 90-100, (1999).

22. V. D. Park \& M. S. Corson, A highly adaptive distributed routing algorithm for mobile wireless networks, In Proceedings of the INFOCOM 97, IEEE, Japan, 3 1405-1413, (1997).

23. C. E. Perkins \& P. Bhagwat, Highly dynamic destination-sequenced distance vector routing (DSDV) for mobile computers. In ACM SIG-COMM, 234-244, (1994).

24. Y-S Yen, H-C Chao, R-S Chang, A Vasilakos, Flooding-limited and multiconstrained QoS multicast routing based on the genetic algorithm for MANETs. Math Comput Model Int J 53(11-12), 2238-2250 (2011).

25. B Berger, M Brady, D Brown, T Leighton, Nearly optimal algorithms and bounds for multilayer channel routing. J ACM 42(2), 500-542 (1995).

26. Vasilakos, W MinYou, CDC: compressive data collection for wireless sensor networks. IEEE Trans Parallel Distrib Syst 26(8), 21882197 (2015).

27. Xi Xu, Rashid Ansari, Ashfaq Khokhar, Athanasios V. Vasilakos, Hierarchical Data Aggregation Using Compressive Sensing (HDACS) in WSNs. ACM Transactions on Sensor Networks (TOSN), 11(3) March (2015).

28. Liu Xiang, Jun Luo, Athanasios V. Vasilakos: Compressed data aggregation for energy efficient wireless sensor networks. SECON, IEEE, USA: 46-54, (2011).

29. AV Vasilakos, L Zhe, S Gwendal, Y Wei, Information centric network: research challenges and opportunities. J Netw Comput Appl 52, 110 (2015).

30. S Zhengguo, Y Shusen, Y Yifan, A Vasilakos, J McCann, L Kin, A survey on the ietf protocol suite for the internet of things: standards, challenges, and opportunities. Wirel Commun IEEE 20(6), 91-98 (2013).

31. X Yang, P Miao, J Gibson, GG Xie, D Ding-Zhu, AV Vasilakos, Tight performance bounds of multihop fair access for mac protocols in wireless sensor networks and underwater sensor networks. Mob Comput IEEE Trans 11(10), 1538-1554 (2012).

32. N Chilamkurti, S Zeadally, A Vasilakos, V Sharma, Cross-layer support for energy efficient routing in wireless sensor networks. Journal of Sensors, Hindawi Publishing Corporation 2009, 9 (2009).

33. Pham, P.P.; Perreau, S. Performance analysis of reactive shortest path and multipath routing mechanism with load balance, INFOCOM, San Francisco, CA, USA, 2003. Twenty-Second Annual Joint Conference of the IEEE Computer and Communications. IEEE Societies, 1 251-259, (2003).

34. M.R. Pearlman, Z.J. Haas, P. Sholander and S.S. Tabrizi,On the impact of alternate path routing for load balancing in mobile ad hoc networks, Proceedings of the ACM MobiHoc, ACM, Boston, 3-10, (2000).

35. S Yin, $X$ Lin, MALB: MANET adaptive load balancing. IEEE Vehicular Technol Conf 4, 2843-2847 (2004).

36. P Merindol, J-J Pansiot, S Cateloin, Improving load balancing with multipath routing, Proceedings of 17 th International Conference on Computer Communications and Networks, 2008 (ICCCN'08, Seattle, Washington, USA, 27-38, (2008).

37. B Nagarjun, L Sathish, S Chaitanya, Md. Ansari, S Tapaswi, Packet count based routing mechanism-a load balancing approach in MANETS. Networked Digital Technologies, Communications in Computer and Information Science 88, 669-675 (2010).

38. B. Sharma, S. Chugh, V. Jain, Energy efficient load balancing approach to improve AOMDV routing in MANET, In 2014 Fourth International Conference on Communication Systems and Network Technologies, IEEE, India, (2014).

39. X Qi, Q Wang, F Jiang, Multi-path routing improved protocol in AODV based on nodes energy. Int J Futur Gener Commun Netw 8(1), 207-214 (2015).

40. S. ljlal Ali Shah, M. Ilyas \& H.T. Mouftah, Pervasive communications handbook, In CRC Press Taylor \& Francis Group, LLC, ISBN-10: 1420051091, ISBN-13: 978-1420051094, (2011). 
41. A Vasilakos, MP Saltouros, AF Atlassis, W Pedrycz, Optimizing QoS routing in hierarchical ATM networks using computational intelligence techniques, Systems, Man, and Cybernetics, Part C: Applications and Reviews, IEEE Transactions (2003).

42. S Thrasyvoulos, R Rao Naveed, T Thierry, O Katia, V Athanasios, Routing for disruption tolerant networks: taxonomy and design. Wirel Netw 16(8), 2349-2370 (2010).

43. Moustafa Youssef, Magdy Abd El-Azim, Mohamed El-Derini, Channel assignment with closeness multipath routing in cognitive networks, Alexandria Engineering Journal, 52(4) 665-670, (2013).

44. Z Yuanyuan, X Kai, L Deshi, AV Vasilakos, Directional routing and scheduling for green vehicular delay tolerant networks. Wirel Netw 19(2), 161173 (2013).

45. Y Liu, N Xiong, Y Zhao, AV Vasilakos, J Gao, Y Jia, Multi-layer clustering routing algorithm for wireless vehicular sensor networks. Commun IET 4(7), 810-816 (2010).

46. W. Pattara-Atikom, P. Krishnamurthy, Quality of service support in IEEE 802.11 Wireless Lan, IEEE Wireless Communications, 10(3) 26-34, (2003).

47. Mahesh K. Marina and Samir R. Das, Ad hoc on-demand multipath distance vector routing, in wireless communications and mobile computing, Wireless Comm, 6 92-96, (2006).

48. H Kai, L Jun, L Yang, AV Vasilakos, Algorithm design for data communications in duty-cycled wireless sensor networks: a survey. Commun Mag IEEE 51(7), 107-113 (2011)

49. Meng, T.; Wu, F.; Yang, Z.; Chen, G.; Vasilakos, A., Spatial reusability-aware routing in multi-hop wireless networks, in Computers, IEEE Transactions on, PP(99) 1-13, (2015).

50. Y Yanjun, C Qing, AV Vasilakos, EDAL: an energy-efficient, delay-aware, and lifetime-balancing data collection protocol for heterogeneous wireless sensor networks. Netw IEEE/ACM Trans 23(3), 810-823 (2015).

51. Zhang, I., Dhurandher, S.K., Anpalagan, A., Vasilakos, A.V., Routing in opportunistic networks. Springer book, New York, (2013).

52. L Liang, S Yuning, Z Haiyang, M Huadong, AV Vasilakos, Physarum optimization: a biology-inspired algorithm for the Steiner Tree Problem in networks. Comput IEEE Trans 64(3), 818-831 (2015).

53. The Network Simulator - ns-2 available on: http://www.isi.edu/nsnam/ns/. September 2015

\section{Submit your manuscript to a SpringerOpen ${ }^{\circ}$ journal and benefit from:}

- Convenient online submission

- Rigorous peer review

- Immediate publication on acceptance

- Open access: articles freely available online

- High visibility within the field

- Retaining the copyright to your article 